\title{
Laterality effects in the processing of melody and timbre
}

\author{
RUSS BOUCHER $†$ and M. P. BRYDEN \\ Department of Psychology, University of Waterloo, Waterloo, Ontario, N2L 3G1, Canada
}

(Received 8 August 1996; accepted 18 April 1997)

\begin{abstract}
Laterality for the processing of melody and timbre was investigated in 64 right-handed non-musicians. In one block of dichotic-listening trials, participants listened for a prespecified target melody, and in a second block they listened for a prespecified target instrument. Females were more accurate on the left ear in the melody task (whereas males tended to show no ear advantage), but there were no significant ear differences in the timbre task for either sex. This supports the idea of a complementary sex-based pattern of lateralization, with males more strongly lateralized for verbal stimuli and females more strongly lateralized for non-verbal stimuli. No relation was observed between lambda measures for the two tasks, suggesting that laterality for melody processing is independent of laterality for timbre processing. (C) 1997 Elsevier Science Ltd
\end{abstract}

Key Words: cerebral asymmetry; dichotic listening; laterality; music.

\section{Introduction}

Music is the most important non-speech sound processed by humans. Like language, music is universal to all humans and specific to humans [20]. Music and language share many other similarities as well [18]. Yet compared to the vast amount of research on laterality for language processing, the number of published articles on laterality for music processing is very small. Further, the literature on dichotic listening using musical stimuli is often contradictory (see [15, 28, 41] for reviews).

Although ear advantages for non-verbal stimuli generally appear to be less robust than those for verbal stimuli [10], the conflicting results often observed in dichotic listening studies using musical stimuli may be due, in part, to the wide range of stimuli and experimental conditions used. The general aim of the present study was to examine laterality for two dimensions of music, melody and timbre, using a more rigorous dichotic listening methodology than had previously been employed with non-verbal stimuli.

\section{Early dichotic listening studies}

In the first dichotic listening study using musical stimuli, Kimura [22] employed a series of trials consisting

$\dagger$ Address for correspondence: 2606 Dunrobin Road, Dunrobin, Ontario, K0A 1T0, Canada; e-mail: rboucher@igs.net. of two melodies presented dichotically, followed by a binaural presentation of four melodies in succession. Participants were then asked to identify the two binaural melodies that were presented dichotically. Using this delayed-recognition paradigm, Kimura found a left-ear advantage (LEA) in a sample of normal right-handed participants.

The finding of an LEA using musical stimuli, along with the earlier observation of a right-ear advantage (REA) for verbal stimuli [21], led to the notion that the left hemisphere was specialized for verbal stimuli and the right hemisphere was specialized for non-verbal stimuli. However, Bartholomeus [3] observed an LEA for letter sequences sung to different melodies, a stimulus that contains an obvious verbal component. As a result, the verbal-non-verbal dichotomy may be too limited to completely account for cerebral lateralization (see [6] for a detailed discussion).

\section{The left hemisphere and music processing}

Some researchers (e.g., [17]) have suggested that the left hemisphere is 'dominant' not for language per se, but rather for temporal information; in a recent review of the literature, Nicholls [26] stated that the left hemisphere appears to be superior for the discrimination of fine temporal events. One aspect of music that may be processed 
by the left hemisphere is timbre, which can be defined as "that attribute of auditory sensation in terms of which a listener can judge that two sounds similarly presented and having the same loudness and pitch are dissimilar" ([1], p. 45). Timbre may be processed in the left hemisphere because temporal information, especially the attack or initial part of a tone, appears to be important for the identification of musical instruments [4, 33]. However, some studies of timbre recognition have failed to show any ear advantage $[14,30,32,35]$, while others have suggested right-hemisphere dominance $[2,13,23,24,31$, 34]. Therefore, although there is good theoretical reason to believe that the processing of timbre may involve the left hemisphere, current data do not support this view.

\section{The role of musical training and processing strategy}

In a very influential study, Bever and Chiarello [5] found an REA for musicians and an LEA for nonmusicians in a melody-recognition task. Bever and Chiarello claimed that musicians were able to organize a melody in terms of the relation of its constituent parts, an analytic strategy eliciting an REA. Non-musicians, on the other hand, tended to focus on the overall melodic contour, a holistic strategy that elicited an LEA. However, this circular argument is rather unconvincing, especially when we consider that the terms analytic and holistic are by no means well defined [19]. Also, it is important to note that the Bever and Chiarello paradigm involved monaural rather than dichotic stimulation, and different factors may be involved here [9].

A more convincing demonstration of the importance of processing strategy in music perception would be to show different ear advantages for different strategy types, while controlling for level of musical training. Peretz and Morais [27] presented non-musicians with a series of dichotic-listening trials using the delayed-recognition paradigm, and instructed participants to attend to and report from either one ear (single focus) or both ears (double focus). An LEA was observed in the single-focus condition, and a slight REA was found in the doublefocus condition, suggesting that ear advantages may be strategy driven rather than stimulus driven.

\section{Methodological concerns}

As can be seen from the preceding discussion, laterality for music processing appears to depend on many factors, including formal domain knowledge and task characteristics [28]. However, the results that have been observed may also be due, in part, to the nature of the response paradigm. Consider that most studies have employed Kimura's [22] delayed-recognition paradigm, in which each dichotic pair is followed by the sequential presentation of four binaural foils. This may not be the optimal response paradigm, because there is evidence that retention of non-verbal sounds starts to decay after about $4 \mathrm{sec}$ [39]. Spreen et al. [36] also found that nonverbal dichotic listening performance declined monotonically as a function of increasing interstimulus interval. Also, Zatorre [40] has shown that performance in the recognition task declines as memory load increases.

Another potential shortcoming of previous studies is that participants are almost always asked to report from both ears, in any order. In a dichotic listening task, responses from the first ear reported are believed to be due to perceptual processes, whereas responses from the second ear are thought to rely more on memory [7]. When participants are free to choose the order of recall, this may have an effect on recall accuracy that is independent of laterality effects [8].

The present study sought to avoid these two potential shortcomings through the use of a variation of the dichotic-monitoring paradigm [16]. In this response paradigm, participants are instructed to listen for a prespecified target, and for each trial are asked to respond 'yes' or 'no' to the question, 'was the target presented?' Number of hits and misses to each ear are computed, as well as the total number of false alarms. Thus, the dichoticmonitoring paradigm is comparable to signal detection. This procedure greatly reduces participants' memory load compared to the delayed-recognition paradigm, and also eliminates possible effects due to order of report.

In the present study, lateralities for melody and timbre were assessed in non-musicians by means of a procedure similar to one used to examine verbal and affective laterality effects [12]. Using stimuli that differed in both melody and timbre, participants were asked to listen for a prespecified target melody, as well as a prespecified target timbre. It was hypothesized that participants would demonstrate an LEA for melody recognition and an REA for timbre recognition. Also, because there may be a wide variability of degree of musical exposure in nonmusicians, data were collected concerning the number of hours per week each participant spent listening to various types of music. This allowed for the investigation of the idea that familiarity with music via listening may also have an effect on overall accuracy, as well as degree of laterality, for a specific task.

\section{Methods}

\section{Subjects}

Sixty-four undergraduate students (32 females and 32 males) at the University of Waterloo participated in the study for pay. All participants were right-handed by self-profession and by the Waterloo Handedness Questionnaire [37]. None of the participants reported ever having taken music lessons, and none played a musical instrument. No participant reported having any hearing difficulties. All subjects gave their informed consent to participate in the study, and were treated according to APA ethical standards. 


\section{Materials}

Stimulus material consisted of four monodic melodies of between seven and 10 notes in length, of $1 \mathrm{sec}$ duration. Each melody was played on four different instruments (clarinet, harp, piano, trumpet), creating 16 different tokens. The melodies were generated using Finale 3.0.1 music notation software on a Macintosh Centris 650 computer. Timbres were selected from the following synthesizers: the piano and trumpet timbres were generated by a Korg Wavestation SR, the harp timbre was generated by a Yamaha DX7-II, and the clarinet timbre was obtained from a Roland D-10. These particular timbres were selected because they were closely related to their respective 'acoustic' instruments. Each melody was paired with each timbre and recorded onto cassette tape using a Yamaha K-222 tape deck. The melodies were excerpts from the following works: a piano transcription of the duettino Là ci darem la mano from W. A. Mozart's Don Giovanni, K. 527; a piano transcription of the aria Non più andrai, farfallone amoroso from W. A. Mozart's Le Nozze di Figaro, K. 492; and the March in D major from J. S. Bach's Notebook of Anna Magdalena Bach (BWV 508-18). The melodies differed in both rhythmic and tonal patterns, and the notes ranged from $\mathrm{C}_{4}(261.63 \mathrm{~Hz})$ to $\mathrm{D}_{5}(587.33 \mathrm{~Hz})$. All the melodies used in the present study were presented at a tempo of 400 beats per minute (bpm), rather than at their normal tempo of approximately $150 \mathrm{bpm}$.

The tokens were equated for length and intensity using Sound-Edit 161.0 sound editing software on a Power Macintosh 7100 computer. Each token was then paired dichotically with every other token that differed in both melody and timbre, creating a total of 144 different trials. Any given melody appeared in the left ear on 36 trials, in the right ear on 36 trials, and was absent on 72 trials; the same was the case for any given timbre. There was a $3-\mathrm{sec}$ interstimulus interval between successive trials and a $10-\mathrm{sec}$ interval after each set of 18 trials. A random sequence of dichotic pairs was created using SoundEdit 161.0 and was then recorded onto cassette tape using a Walkman Professional WM-D60. These randomized dichotic pairs were presented at an average intensity of $75 \mathrm{~dB}$ on a Sony TC-R303 tape deck through JVC HA-D500 headphones.

\section{Procedure}

Participants were tested on an individual basis in a soundproof room, and were presented with two blocks of dichotic listening trials. In one block of trials, participants were instructed to listen for a prespecified target melody, and in a second block of trials they were instructed to listen for a prespecified target instrument. The specific target and order of presentation were counterbalanced between participants. In order to control for possible minor differences between channels, half of the participants were tested with the headphones in one orientation and half with the reverse orientation. All the above counterbalancing measures were complete for each sex.

After being given a brief description of the nature of the dichotic listening procedure, each participant was presented with a target, and was then told that in the present study there were four different melodies, each played on four different instruments. The instruments were identified as a clarinet, a harp, a piano and a trumpet. This was followed by a binaural presentation of the 16 tokens in random order. Participants were instructed that they would hear their target four times, and were told to indicate the presence of their target by circling the number of the corresponding trial on a response sheet. If a participant had trouble identifying his or her target, the experimenter presented the target a second time, followed by a second presentation of the 16 tokens. This was necessary in $31 \%$ of the cases using a melody target, and in $14 \%$ of the cases using an instrument target.

When the experimenter was confident that the participant could discriminate her or his target from the distractors in the binaural presentation, the participant was presented with the first block of dichotic listening trials. The participant was told to indicate the presence or absence of the target on each trial by circling either 'yes' or 'no' on a response sheet. When the participants had finished the first block of trials (which took approximately $20 \mathrm{~min}$ to complete), they were administered the Waterloo Handedness Questionnaire [37] and a music listening questionnaire. The entire testing procedure was then repeated, and participants who had just been given a target melody were now given a target instrument, and vice versa. At the end of the second block of dichotic listening trials, participants were informed as to the nature of the study via a verbal debriefing, and were then paid for their participation. The possibility that performance on the melody task was facilitated by previous familiarity of the target melody was checked in two ways: during the debriefing session, participants were asked if they recognized any of the four melodies as being familiar; none of them did. Also, almost all participants reported on the music listening questionnaire that they did not listen to classical music.

\section{Results}

\section{Overall analysis}

An initial analysis of variance of the number of correct responses, with sex, task and order as between-subjects variables, and ear of presentation as a within-subjects variable, revealed a significant task by ear interaction $[F(1,60)=5.92, P=0.018]$, which suggests that different ear advantages appeared on the two tasks. However, this was qualified by the presence of a sex by task by ear interaction $[F(1,60)=4.54, P=0.037]$. Overall, there was an LEA for the melody task and a non-significant REA for the timbre task. In males, this effect was trivially small and non-significant, whereas in females there was a significant LEA for the melody task and non-significant REA for the timbre task. Males tended to be more accurate overall, whereas females tended to show larger ear differences (see Table 1).

There was also a significant task by order interaction $[F(1,60)=9.84, P=0.003]$. Note that the task by order interaction does not interact with ear of presentation. As can be seen in Table 2, participants were more accurate in both ears when they performed a task first than when they performed it second.

\section{Melody task}

In order to determine whether these effects were consistent across individual melodies and timbres, separate analyses were carried out on the melody and timbre data. These analyses were performed because it was not possible to examine the effects of individual melodies and timbres in the overall analysis. An analysis of variance of the melody data, with sex, order and melody as between- 
Table 1. Percent correct as a function of sex, task and ear of presentation

\begin{tabular}{lccccccc}
\hline & \multicolumn{3}{c}{ Task } \\
\cline { 2 - 4 } & \multicolumn{3}{c}{ Melody } & & \multicolumn{3}{c}{ Timbre } \\
\cline { 2 - 4 } \cline { 5 - 7 } & Left & Right & False alarms & & Left & Right & False alarms \\
\hline Males & 70.49 & 69.97 & 20.27 & & 92.53 & 92.71 & 4.08 \\
Females & 67.53 & 58.07 & 21.70 & & 87.24 & 88.19 & 3.86 \\
\hline
\end{tabular}

Table 2. Percent correct as a function of order, task and ear of presentation*

\begin{tabular}{|c|c|c|c|c|c|c|}
\hline & \multicolumn{6}{|c|}{ Task } \\
\hline & \multicolumn{3}{|c|}{ Melody } & \multicolumn{3}{|c|}{ Timbre } \\
\hline & Left & Right & False alarms & Left & Right & False alarms \\
\hline MT & 74.22 & 69.44 & 19.97 & 87.07 & 86.37 & 4.95 \\
\hline TM & 63.80 & 58.59 & 22.01 & 92.71 & 94.53 & 2.99 \\
\hline
\end{tabular}

*MT, melody task first; TM, timbre task first.

subjects variables, and ear of presentation as a withinsubjects variable, yielded a main effect of ear $[F(1,48)=5.14, P=0.028]$. The main effect of ear was qualified by a sex by ear interaction $[F(1,48)=4.12$, $P=0.048$. Males tended to show no ear advantage, whereas females showed a significant LEA. There was also a main effect of order $[F(1,48)=4.47, P=0.040]$. Participants were more accurate in both ears when they performed the melody task first than when they performed it second. The main effect of melody did not even approach significance $[F(3,48)=0.57 P=0.637]$, neither did the melody by ear interaction $[F(3,48)=0.24$, $P=0.871$ ]. As can be seen in Table 3 , all four melodies tended to elicit an LEA of roughly the same size.

Although the overall false alarm rate for the melody task was over $20 \%$, the hit rate was much higher, suggesting that participants were in fact able to do the task,

Table 3. Laterality effects as a function of target

\begin{tabular}{lcccc}
\hline & \multicolumn{4}{c}{ Percent correct } \\
\cline { 2 - 5 } & Left ear & Right ear & Difference & False alarms \\
\hline Melody 1 & 69.62 & 61.98 & -7.64 & 17.71 \\
Melody 2 & 64.76 & 62.33 & -2.43 & 15.97 \\
Melody 3 & 74.65 & 69.44 & -5.21 & 25.09 \\
Melody 4 & 67.01 & 62.33 & -4.68 & 25.17 \\
All melodies & 69.01 & 64.02 & -4.99 & 20.99 \\
Clarinet & 86.98 & 83.51 & -3.47 & 4.08 \\
Harp & 89.06 & 91.32 & +2.26 & 7.73 \\
Piano & 93.40 & 95.83 & +2.43 & 0.26 \\
Trumpet & 90.10 & 91.15 & +1.05 & 3.82 \\
All timbres & 89.89 & 90.45 & +0.56 & 3.97 \\
\hline
\end{tabular}

although with some difficulty. None of the other main effects or interactions were significant $(P>0.07$ in all cases).

\section{Timbre task}

Analysis of the timbre data alone, with sex, order and timbre as between-subjects variables, and ear of presentation as a within-subjects variable, showed only a main effect of order $[F(1,48)=6.81, P=0.012]$. Participants were more accurate in both ears when they performed the timbre task first than when they performed it second. The main effect of timbre did not even approach significance $[F(3,48)=2.11, P=0.111]$, neither did the timbre by ear interaction $[F(3,48)=0.97, P=0.416]$. None of the other main effects or interactions were significant ( $P>0.07$ in all cases). Although there was a slight REA for three of the four instruments, overall there was only a marginal ear difference (see Table 3 ).

\section{Correction for overall accuracy}

In both the melody and timbre tasks, overall accuracy was negatively correlated with false alarm rate. As can be seen in Table 3, the overall hit rate for the timbre task was very high and the false alarm rate was very low, with the opposite pattern present in the melody task. In order to obtain laterality measures that were independent of overall accuracy, lambda values were computed for each participant for both tasks. The lambda measure of laterality [11] is based on the log-odds ratio, and in the present study was computed as $\ln \left[\left(H_{\mathrm{R}} \times M_{\mathrm{L}}\right) /\left(M_{\mathrm{R}} \times H_{\mathrm{L}}\right)\right]$, where $H_{\mathrm{R}}$ and $H_{\mathrm{L}}$ are the number of hits to the left ear and 
right ear, respectively, and $M_{\mathrm{L}}$ and $M_{\mathrm{R}}$ are the number of misses to the left ear and right ear, respectively. Thus, negative lambda values indicate an LEA, whereas positive lambda values indicate an REA. An analysis of variance on the lambda values, with sex and order as betweensubjects variables, and task as a within-subjects variable, revealed a significant main effect of task $[F(1,60)=4.52$, $P=0.038]$. Overall, there was an LEA for the melody task, but no ear advantage for the timbre task. This substantiates the original analysis, while ensuring that the differences between melody and timbre are not due to accuracy differences. A separate analysis of the melody lambda values, with sex, order and melody as betweensubjects variables, showed a significant main effect of sex $[F(1,60)=5.48, P=0.023]$. None of the other main effects or interactions were significant $(P>0.241$ in all cases). Analysis of the timbre lambda values alone, with sex, order and timbre as between-subjects variables, did not reveal any significant main effects or interactions $(P>0.234$ in all cases).

\section{Overall performance and musical familiarity}

It is possible that some of the individual differences in ear advantages may be attributable to differences in familiarity with music (e.g., [5]). In order to investigate the role of musical familiarity (as indicated by the number of hours per week spent listening to music) on performance in the melody and timbre tasks, two separate regression analyses were performed. For each task, the number of correct responses to each ear was summed and then used as the dependent variable in a linear regression analysis, with number of hours per week spent listening to music as the independent variable. There was a positive relation of marginal significance between listening and overall performance on the melody task $(r=0.242$, $b=0.387, P=0.054$ ), accounting for approximately $5.9 \%$ of the variance. Participants who spent more time listening to music tended to be more accurate on the melody task than those who did not listen to music as often. In the timbre task, there was essentially no relation between listening and overall performance $(r=-0.049$, $b=-0.045, P=0.701$ ). Performance on the two tasks was essentially uncorrelated $(r=0.003)$. Males and females did not differ significantly in terms of the relation between musical familiarity and performance on either task.

In a similar fashion, two regression analyses were performed in order to investigate whether the size of the observed laterality effects in each task were related to musical familiarity. There was no relation between lambda values and listening for the melody task ( $r=0.018, b=-0.002, P=0.886$ ) or for the timbre task $(r=0.033, \quad b=-0.004, \quad P=0.794)$. Therefore, participants who listened to music more often did not tend to show larger ear differences than those who did not listen to music as often.
Table 4. Contingency table of lambda values for melody and timbre recognition tasks*

\begin{tabular}{lccccc}
\hline & & \multicolumn{4}{c}{ Melody recognition } \\
\cline { 3 - 6 } & & LEA & NEA & REA & Total \\
\hline Timbre recognition & LEA & 10 & 2 & 11 & 23 \\
& NEA & 11 & 0 & 6 & 17 \\
& REA & 12 & 2 & 10 & 24 \\
& Total & 33 & 4 & 27 & 64 \\
\hline
\end{tabular}

*LEA, left ear advantage; NEA, no ear advantage; REA, right ear advantage.

Lambda values for the two tasks were weakly correlated ( $r=0.144, P=0.258$ ), although it is important to note that this correlation is constrained by the reliability of the two tasks. However, as shown in Table 4, laterality for one task appears to be relatively independent of laterality for the other task. For example, given that a participant showed an LEA for melody recognition, the odds of him or her showing an LEA or an REA for timbre recognition are approximately equal (odds ratio $=1.32$, excluding cases showing no ear advantage for either task).

\section{Discussion}

The results of the present study can be summarized as follows: (i) there was a significant task by ear interaction, indicating an LEA for melody recognition but no ear advantage for timbre recognition (although there was a very small REA for three of the four instruments); (ii) females were more likely to show an LEA for the melody task, whereas males showed no ear advantage but tended to be more accurate overall; (iii) participants were more accurate in both ears when they performed a task first than when they performed it second; (iv) higher levels of musical familiarity were associated with increased overall accuracy on the melody task, but not on the timbre task; (v) there was no relation observed between musical familiarity and degree of laterality on either task; and (vi) there was no relation observed between overall accuracy and degree of laterality on either task, or between degree of laterality for the two tasks.

The overall emergence of an LEA for melody recognition supports previous research suggesting that the right hemisphere is superior for the processing of musical stimuli $[22,35,36]$. No significant REA was observed for timbre recognition, but recall that previous studies of a similar nature also failed to find an ear advantage [14, $30,35]$. The results of the present study also highlight the importance of processing strategy [27]. The same stimulus set elicited a significant LEA for melody and the hint of an REA for timbre, just by changing the response category. However, because overall accuracy for the timbre task was approximately $90 \%$, it is quite possible that there was a ceiling effect. 
No relation was observed between lambda values for the two tasks, which suggests that laterality for melody recognition is independent of laterality for timbre recognition. Participants who showed an LEA on the melody task were not more likely to show an REA for the timbre task (and vice versa), as one might expect. Generally, because most people show an REA for verbal stimuli and an LEA for non-verbal stimuli, it appears as if lateralities for the two types of material are related. However, Bryden [9] has argued that the majority of right handers may show a left-hemisphere superiority for language and a right-hemisphere superiority for nonverbal processes solely by chance. Thus, the results of the present study would support Bryden's [9] statement that "the observation in an individual that one hemisphere is specialized for a particular function implies nothing about the functions of the other hemisphere in that individual" (p. 274).

\section{Sex differences in music processing?}

The only paper of which the present authors are aware that has reported a sex difference for melody recognition is that of Piazza [29], who found a significant LEA for right-handed females but not for right-handed males. These results are similar to those observed in the present study: namely, that females appear to be more lateralized than males for melody recognition. It is interesting to note a similarity between this study and studies of verbal dichotic listening, in which males tend to show a greater REA than females [10, 38]. This supports the idea of a complementary sex-based pattern of auditory lateralization, with males more strongly lateralized for verbal stimuli and females more strongly lateralized for nonverbal stimuli $[9,29]$ (cf. [38]).

\section{Memory and attentional considerations}

A small percentage of the variability in overall accuracy on the melody task was observed to be due to musical familiarity as measured by participants' recollection of amount of time spent listening to music. One explanation for these results might be that people who listen to music on a regular basis can remember music better (perhaps via rehearsal), and are capable of retaining a target melody in memory for a longer period of time. Also, although it is important to note that the nature of the two recognition tasks is somewhat different, it is quite possible that memory demands for the two tasks may not have been comparable. This could account, in part, for the absence of a correlation between overall performance on the two tasks.

Recall that the dichotic-monitoring paradigm was used in the present study in order to reduce memory load and avoid bias resulting from order of report. However, because participants were not required to indicate the ear in which they heard (or thought they heard) the target, the possibility that participants biased their attention towards one ear cannot be ruled out. As a result, in some cases the degree and/or direction of the ear difference may be due to attentional factors as well as to laterality effects. Such attentional strategies could be reduced by using a precuing technique [25]. There are two types of spatial cues that are commonly used. An exogenous cue is presented in the location of the stimulus to follow (e.g., a tone in the right ear), while an endogenous cue indicates the location of the stimulus to follow, but from a spatially neutral location (e.g., the word 'right' presented binaurally). Therefore, the precuing technique should provide a measure of laterality that is less likely to be influenced by attentional factors.

Acknowledgements - This paper is based on a Master's thesis by the first author submitted to the University of Waterloo, July 1996. The authors are grateful to David Huron for many helpful discussions, to Barbara Bulman-Fleming and Jennifer Stolz for comments on the manuscript, to Peter Hatch for graciously providing access to his MIDI studio, and to Patricia Connelly for technical assistance. Portions of this article were presented at the 25th Meeting of the International Neuropsychological Society, February 1997, Orlando, FL, U.S.A. This research was supported by a grant to $M$. P. Bryden from the Natural Sciences and Engineering Research Council of Canada.

\section{References}

1. American Standards Association. American Standard Acoustical Terminology S1-1 1960. American Standards Association, New York, 1960.

2. Auzou, P., Eustache, F., Etevenon, P., Platel, H., Rioux, P., Lambert, J., Lechevalier, B., Zarifian, E. and Baron, J. C., Topographic EEG activations during timbre and pitch discrimination tasks using musical sounds. Neuropsychologia, 1995, 33, 25-37.

3. Bartholomeus, B., Effects of task requirements on ear superiority for sung speech. Cortex, 1974, 10, 215-223.

4. Berger, K. W., Some factors in the recognition of timbre. Journal of the Acoustical Society of America, 1964, 36, 1888-1891.

5. Bever, T. G. and Chiarello, R. J., Cerebral dominance in musicians and nonmusicians. Science, 1974 , $185,537-539$.

6. Bradshaw, J. L. and Nettleson, N. C., The nature of hemispheric specialization in man. The Behavioral and Brain Sciences, 1981, 4, 51-91.

7. Broadbent, D. E., Immediate memory and simultaneous stimuli. Quarterly Journal of Experimental Psychology, 1957, 4, 1-11.

8. Bryden, M. P., Strategy effects in the assessment of hemispheric asymmetry. In Strategies of Information Processing, ed. G. Underwood. Academic Press, London, 1978, pp. 117-149.

9. Bryden, M. P., Laterality: Functional Asymmetry in the Intact Brain. Academic Press, New York, 1982. 
10. Bryden, M. P., An overview of the dichotic listening procedure and its relation to cerebral organization. In Handbook of Dichotic Listening: Theory, Methods and Research, ed. K. Hugdahl. John Wiley, New York, 1988, pp. 1-43.

11. Bryden, M. P. and Sprott, D. A., Statistical determination of degree of laterality. Neuropsychologia, 1981, 19, 571-581.

12. Bulman-Fleming, M. B. and Bryden, M. P., Simultaneous verbal and affective laterality effects. $\mathrm{Neu}$ ropsychologia, 1994, 32, 787-797.

13. Chobar, K. L. and Brown, J. W., Phoneme and timbre monitoring in left and right cerebrovascular accident patients. Brain and Language, 1987, 30, 278284.

14. Darwin, C. J., Auditory Perception and Cerebral Dominance. Unpublished doctoral dissertation, University of Cambridge, 1969.

15. Gates, A. and Bradshaw, J. L., The role of the cerebral hemispheres in music. Brain and Language, $1977,4,403-431$.

16. Geffen, G. and Caudrey, D., Reliability and validity of the dichotic monitoring test for language laterality. Neuropsychologia, 1981, 19, 413-423.

17. Halperin, Y., Nachson, I. and Carmon, A., Shift of ear superiority in dichotic listening to temporally patterned nonverbal stimuli. Journal of the Acoustical Society of America, 1973, 53, 46-50.

18. Handel, S., Listening: An Introduction to the Perception of Auditory Events. MIT Press, Cambridge, MA, 1989.

19. Hellige, J. B., Hemispheric Asymmetry: What's Right and What's Left. Harvard University Press, Cambridge, MA, 1993.

20. Herskovits, M., Man and His Works. Knopf, New York, 1960.

21. Kimura, D., Cerebral dominance and the perception of verbal stimuli. Canadian Journal of Psychology, 1961, 15, 166-171.

22. Kimura, D., Left-right differences in the perception of melodies. Quarterly Journal of Experimental Psychology, 1964, 16, 355-358.

23. Mazziotta, J. C., Phelps, M. E., Carson, R. E. and Kuhl, D. E., Tomographic mapping of cerebral metabolism: auditory stimulation. Neurology, 1982, 32, 921-937.

24. Milner, B., Laterality effect in audition. In Interhemispheric Relations and Cerebral Dominance, ed. V. Mountcastle. John Hopkins Press, Baltimore, 1962, pp. 177-195.

25. Mondor, T. A. and Bryden, M. P., On the relation between auditory spatial attention and auditory perceptual asymmetries. Perception and Psychophysics, 1992, 52, 393-402.
26. Nicholls, M. E. R., Temporal processing asymmetries between the cerebral hemispheres: evidence and implications. Laterality, 1996, 1, 97-138.

27. Peretz, I. and Morais, J., Task determinants of ear differences in melody processing. Brain and Cognition, 1983, 2, 313-330.

28. Peretz, I. and Morais, J., Determinants of laterality for music: towards an information processing account. In Handbook of Dichotic Listening: Theory, Methods and Research, ed. K. Hugdahl. John Wiley, New York, 1988, pp. 323-358.

29. Piazza, D. M., The influence of sex and handedness in the hemispheric specialization of verbal and nonverbal tasks. Neuropsychologia, 1980, 18, 163-176.

30. Prior, M. and Troop, G. A., Processing of timbre and rhythm in musicians and non-musicians. Cortex, 1988, 24, 451-456.

31. Rastatter, M. P. and Gallagher, A. J., Reaction-times of normal subjects to monaurally presented verbal and tonal stimuli. Neuropsychologia, 1982, 20, 465473.

32. Rushford, K. and Murray, D., Left-right differences in the processing of instrument tone segments. Council for Research in Music Education, 1977, 52, 1-6.

33. Saldanha, E. L. and Corso, J. F., Timbre cues and the identification of musical instruments. Journal of the Acoustical Society of America, 1964, 36, 20212026.

34. Samson, S. and Zatorre, R. J., Contribution of the right temporal lobe to musical timbre discrimination. Neuropsychologia, 1994, 32, 231-240.

35. Spellacy, F., Lateral preferences in the identification of patterned stimuli. Journal of the Acoustical Society of America, 1970, 47, 574-578.

36. Spreen, O., Spellacy, F. J. and Reid, J. R., The effect of interstimulus interval and intensity on ear asymmetry for nonverbal stimuli in dichotic listening. Neuropsychologia, 1970, 8, 245-250.

37. Steenhuis, R. E. and Bryden, M. P., Different dimensions of hand preference that relate to skilled and unskilled activities. Cortex, 1989, 25, 289-304.

38. Voyer, D., On the magnitude of laterality effects and sex differences in functional brain lateralities. Laterality, 1996, 1, 51-83.

39. Wolf, C. G., The processing of fundamental frequency in a dichotic matching task. Brain and Language, 1977, 4, 70-77.

40. Zatorre, R. J., Recognition of dichotic melodies by musicians and nonmusicians. Neuropsychologia, $1979,17,607-617$.

41. Zatorre, R. J., Musical perception and cerebral function: a critical review. Music Perception, 1984, 2, 196221 . 